\title{
Eye Redness
}

National Cancer Institute

\section{Source}

National Cancer Institute. Eye Redness. NCI Thesaurus. Code C118724.

The prominence of the superficial vessels of the sclera or conjunctiva resulting in a red or pink appearance. 